\title{
TBC bond coat-top coat interface roughness: influence on fatigue life and modelling aspects
}

Robert Eriksson, Sören Sjöström, Håkan Brodin, Sten Johansson, Lars Östergren and Xin-Hai $\mathrm{Li}$

\section{Linköping University Post Print}

\section{Tweet}

N.B.: When citing this work, cite the original article.

Original Publication:

Robert Eriksson, Sören Sjöström, Håkan Brodin, Sten Johansson, Lars Östergren and Xin-Hai $\mathrm{Li}, \mathrm{TBC}$ bond coat-top coat interface roughness: influence on fatigue life and modelling aspects, 2013, Surface \& Coatings Technology, (236), 230-238.

http://dx.doi.org/10.1016/j.surfcoat.2013.09.051

Copyright: Elsevier http://www.elsevier.com/

Postprint available at: Linköping University Electronic Press http://urn.kb.se/resolve?urn=urn:nbn:se:liu:diva-96811 


\title{
TBC bond coat-top coat interface roughness: influence on fatigue life and modelling aspects
}

\author{
Robert Eriksson ${ }^{\mathrm{a}, *}$, Sören Sjöström ${ }^{\mathrm{a}}$, Håkan Brodin ${ }^{\mathrm{b}, \mathrm{a}}$, Sten Johansson ${ }^{\mathrm{a}}$, \\ Lars Östergren ${ }^{c}$, Xin-Hai Li ${ }^{b}$ \\ ${ }^{a}$ Division of Engineering Materials, Department of Management and Engineering, \\ Linköpings universitet, SE-58183 Linköping, Sweden \\ ${ }^{b}$ Siemens Industrial Turbomachinery AB, SE-61283 Finspong, Sweden \\ ${ }^{c}$ GKN Aerospace Engine Systems, SE-46181 Trollhättan, Sweden
}

\begin{abstract}
Thermal barrier coatings (TBCs), when used in gas turbines, may fail through thermal fatigue, causing the ceramic top coat to spall off the metallic bond coat. The life prediction of TBCs often involves finite element modelling of the stress field close to the bond coat/top coat interface and thus relies on accurate modelling of the interface. The present research studies the influence of bond coat/top coat interface roughness on the thermal fatigue life of plasma sprayed TBCs. By using different spraying parameters, specimens with varying interface roughness were obtained. During thermal cycling it was found that higher interface roughness promoted longer thermal fatigue life. The interfaces were characterised by roughness parameters, such as $R a, R q$ and $R \Delta q$, as well as by autocorrelation, material ratio curves and slope distribution. The variation of spray parameters was found to affect amplitude parameters, such as $R a$, but not spacing parameters, such as $R S m$. Three different interface geometries were tried for finite element crack growth simulation: cosine, ellipse and triangular shape. The cosine model was found to be an appropriate interface model and a procedure for obtaining the necessary parameters, amplitude and wavelength, was suggested. The positive effect of high roughness on life was suggested to be due to a shift from predominantly interface failure, for low roughness, to predominantly top coat failure, for high roughness.
\end{abstract}

Keywords: thermal barrier coating, TBC, thermal cycling fatigue, interface, roughness, Ra 


\section{Introduction}

Thermal barrier coating (TBC) systems are coating systems which includes a thermally insulating ceramic top coat (TC), and an oxidation resistant metallic bond coat (BC) [1-4]. The purpose of such coating systems is to lower the temperature of the underlying substrate. When used in gas turbines, TBC systems can prolong the life of structural parts, as well as increase the gas turbine efficiency by enabling higher combustion temperatures [1-4]. TBC systems, consequently, have found great use in gas turbine technology.

Due to their importance in gas turbine technology, accurate life prediction of TBC systems is crucial. TBC systems may fail during operation due to thermal fatigue caused by thermal loads which arise from the difference in coefficient of thermal expansion (CTE) of the ceramic top coat and the metallic bond coat [5-7]. Failure of TBCs often occurs close to the BC/TC interface where fatigue cracks grow under a stress field influenced by various parameters: the $\mathrm{BC}-\mathrm{TC}$ mismatch in $\mathrm{CTE}$, the $\mathrm{BC} / \mathrm{TC}$ interface roughness and the growing layer of thermally grown oxides (TGO) that form in the interface [5-7].

Several approaches to TBC life prediction exist, one of which is a mechanical approach, typically involving finite element (FE) modelling of stresses close to the $\mathrm{BC} / \mathrm{TC}$ interface $[5,6]$. The importance of the near-interface stress field in TBC life prediction is illustrated by the large number of papers which deal with this [5-40]. Many researchers thus turn their attention to the stress state in the $\mathrm{BC} / \mathrm{TC}$ interface looking for explanations of $\mathrm{TBC}$ failure.

To accurately establish the near-interface stresses by FE modelling, care must be taken to the modelling of the $\mathrm{BC} / \mathrm{TC}$ interface. The cosine wave has emerged as a common interface model with amplitudes and wavelengths often in the intervals $5-20 \mu \mathrm{m}$ and $20-150 \mu \mathrm{m}$ respectively $[7-11,14,17$, 18, 20-23, 26-31, 33-36, 38-40]. However, in-depth studies of the actual $\mathrm{BC} / \mathrm{TC}$ interface roughness, and its influence on fatigue life, are still rare. More importantly, the plausibility of the cosine interface model has never been evaluated.

Among the few studies on the influence of $\mathrm{BC} / \mathrm{TC}$ interface roughness on fatigue life can be mentioned Vassen et al. [38] who studied TBC coated

*Corresponding author. Tel.: +46 13 284410; fax: +46 13 282505. E-mail address: robert.eriksson@liu.se 
specimens with a $\mathrm{BC} / \mathrm{TC}$ interface roughness of $R a=4.9-7.2 \mu \mathrm{m}$; the different roughness were obtained by using different feedstock powder sizes during spraying. The thermal cycling was interrupted prior to failure and cross-sections revealed that lower roughness gave longer cracks close to the BC/TC interface. Furthermore, Casu et al. [17] performed FE crack growth modelling of an interface geometry derived from an actual BC/TC interface through autocorrelation-based techniques. Casu et al. [17], too, studied specimens sprayed with different powder sizes and found features in the $\mathrm{BC} / \mathrm{TC}$ interface which could be related to process parameters.

In the present study, TBC coated specimens with various interface roughness were thermally cycled until failure and then cross-sectioned. Image analysis was used to characterise the $\mathrm{BC} / \mathrm{TC}$ interface by the means of various surface roughness parameters. The interface roughness parameters were then used to derive several interface models which were evaluated through FE modelling of a crack growing close to the interface. The experimental and $\mathrm{FE}$ results were used to establish a robust method for transforming the rather chaotic $\mathrm{BC} / \mathrm{TC}$ interface into a representative interface model. The present study hence provides knowledge about appropriate interface models that can be used for FE modelling of TBCs.

\section{Roughness parameters}

To facilitate the reading of this paper, a few of the surface roughness parameters are defined and explained here. For a more extensive explanation of surface roughness measurements, refer to literature such as Gadelmawla et al. [41]. Definitions of surface roughness parameters and related information can also be found in various standards [42-44].

The $2 \mathrm{D}$ mathematical representation of the interface is referred to as the profile. After profile acquisition, for example by a profilometer, the measured profile is separated into longwave and shortwave components by the use of a Gaussian filter. The filtering is controlled through the cut-off wavelengths $\lambda_{f}>\lambda_{c}>\lambda_{s}$ such that: $\mathrm{P}$-profile $>\lambda_{s}, \lambda_{f}>\mathrm{W}$-profile $>\lambda_{c}$ and $\lambda_{c}>\mathrm{R}$-profile $>\lambda_{s}$. The R-profile, roughness profile, is the one of interest here.

$R c$ is the mean value of the profile elements height, $z t_{i}$, see Fig. 1.

$$
R c=\frac{1}{m} \sum_{i=1}^{m} z t_{i}
$$


$R a$ is the arithmetic mean value of the absolute z-coordinates.

$$
R a=\frac{1}{L} \int_{0}^{L}|z(x)| d x
$$

$R q$ is the root mean square value of the z-coordinates.

$$
R q=\sqrt{\frac{1}{L} \int_{0}^{L} z^{2}(x) d x}
$$

$R S m$ is the mean value of the profile element widths, $x s_{i}$, see Fig. 1.

$$
R S m=\frac{1}{m} \sum_{i=1}^{m} x s_{i}
$$

$R d q$ is the root mean square value of the derivative of the profile.

$$
R \Delta q=\sqrt{\frac{1}{L} \int_{0}^{L}\left(\frac{d z}{d x}\right)^{2} d x}
$$

\section{Experimental}

\subsection{Materials}

The studied TBC systems consisted of $200 \mu \mathrm{m}$ of vacuum plasma sprayed (VPS) bond coat and $350 \mu \mathrm{m}$ of atmospheric plasma sprayed (APS) 7\%yttria partially-stabilised zirconia deposited on substrates of Haynes 230. The specimen substrates had a rectangular shape, 30x50 mm, and were cut from $5 \mathrm{~mm}$ thick sheet material. The bond coat consisted of $\mathrm{Ni}-25 \mathrm{Cr}-$ 5Al-2.6Si-1Ta-0.6Y, all in wt.\%. Four specimens with different BC/TC interfaces were obtained by using feedstock powders of varying particle size during spraying of the bond coat. The consequence of varying the powder size was specimens with varying $\mathrm{BC} / \mathrm{TC}$ interface roughness; cross-sections of the specimens are shown in order of increasing interface roughness in Fig. 2 a)-d). The specimens will, in this paper, be referred to as R-low, Rmedium-1, R-medium-2 and R-high in accordance with the order in which they appear in Fig. 2, i.e. the R-low specimen had the lowest roughness, the R-medium-1 and R-medium-2 specimens had intermediate roughness, and the R-high specimen had the highest roughness. 


\subsection{Thermal cycling and microscopy}

The specimens were thermally cycled until failure in a thermal cycling fatigue (TCF) furnace. One cycle consists of heating to $1100{ }^{\circ} \mathrm{C}$ followed by a high temperature dwell time of $60 \mathrm{~min}$. The specimens are then moved out of the furnace and cooled by compressed air for 10 min thereby reaching a minimum temperature of $100{ }^{\circ} \mathrm{C}$, measured in the centre of the substrate. Of the $60 \mathrm{~min}$ dwell time approximately $5 \mathrm{~min}$ are required to reach maximum temperature in the centre of the substrate. The specimens were cycled as-manufactured with no preparation of TBC edges. Thermal cycling was performed until failure, where failure was considered to have occurred when $20 \%$ of the top coat had delaminated; delamination typically occurred from the specimen edges.

After failure, the specimens were cross-sectioned and mounted for microscopy. Prior to cutting, the specimens were epoxy infiltrated in vacuum. Electron microscopy was performed witch a Hitachi SU-70 field emission gun scanning electron microscope (SEM) equipped with an EDS detector from Oxford Instruments. Before analysis in SEM, the specimens were coated with approximately $25 \mathrm{~nm}$ carbon to decrease charging.

\subsection{Surface roughness measurements}

The surface roughness measurements were performed on cross-sectioned specimens by image analysis using Matlab. The image analysis procedure was calibrated against profilometer results for a BC coated specimen (no top coat). Good agreement with a profilometer was accomplished by simulating a profilometer's stylus tip mathematically as a semi-circle 'dragging' along the interface profile.

The exact procedure for interface roughness measurement, by image analysis, has been described elsewhere [45] and is only briefly outlined here. The procedure includes:

1. Image acquisition by light-optic microscopy.

2. Thresholding the obtained grayscale image to get a binary image. The thresholding will render the bond coat white and the top coat and TGO black; i.e. the measured interface is the bond coat/TGO interface.

3. Acquisition of the roughness profile from the binary image by 'dragging' a semi-circle along the interface to simulate a stylus tip.

4. Separation of the profile into $\mathrm{P}-, \mathrm{W}$ - and R-profiles using Gaussian filtering. 
5. Establishing the mean line of the roughness profile.

6. Calculation of the roughness parameters from the roughness profile.

The $\lambda_{s}$ cut-off wavelength is usually set to a value of the order of the dimension of the stylus tip used. The simulated stylus tip had a radius of $5 \mu \mathrm{m}$ and the $\lambda_{s}$ was set to $10 \mu \mathrm{m}$. The studied specimens had substrates made from wrought sheet material with a noticeable waviness; the waviness wavelength was previously estimated to $\sim 650 \mu \mathrm{m}$ [7]. Setting the $\lambda_{c}$ to a value in that order of magnitude would make the R-profile less dependent on the substrate and can be assumed to capture mainly the interface characteristics imposed by the plasma spray process, which was desirable here. In order to establish a suitable $\lambda_{c}$ cut-off wavelength, the influence of $\lambda_{c}$ on $R a$ and $R S m$ was studied. The $\lambda_{c}$ was varied in the interval $400-1000$ $\mu \mathrm{m}$. The $R a$ and $R S m$ values did vary with the cut-off wavelength in this interval, but the variation was reasonable small: 10-15 $\mu$ m change in $R S m$ and $0.3-0.4 \mu \mathrm{m}$ change in $R a$ per $100 \mu \mathrm{m}$ change in $\lambda_{c}$. Since the exact number chosen in the interval $400-1000 \mu \mathrm{m}$ had little effect on the results, the cut-off wavelength was set to $800 \mu \mathrm{m}$ which is close to $650 \mu \mathrm{m}$ and is a commonly used value.

In total, $52 \mathrm{~mm}$ of the interface was studied, divided in 65 sampling lengths. All images used for surface roughness measurements were obtained by light-optic microscopy. The chosen magnification gave images with a resolution of $1.075 \mu \mathrm{m} /$ pixel which was found to be sufficient [45].

\section{Results and Discussion}

\subsection{TCF life and TGO formation}

A fractured specimen (with a TCF life of $\sim 700$ cycles) is shown in Fig. 3. The fractured occurred in, or close to, the TGO/TC interface; this fracture behaviour was common for all specimens. The specimens had largely different TCF lives: the R-low specimen failed at $\sim 500$ cycles, the both R-medium specimens at $\sim 700 \pm 13$ cycles and the R-high specimen failed at 850 cycles. The cycles to failure thus increased with increasing interface roughness.

Good adhesion of APS TBCs to the bond coat is often described as achieved mainly by mechanical anchoring [46] and a higher roughness may therefore promote higher adhesion. The influence of roughness on thermal fatigue life observed here also agrees with the findings of Vassen et al. [38] 
who found that lower roughness gave longer cracks close to the $\mathrm{BC} / \mathrm{TC}$ interface.

The oxidation and microstructural degradation of these TBC systems have been reported elsewhere [7] and is only briefly summarised here: The four specimens all showed similar oxide compositions and oxidation kinetics; the formed TGO (at failure) consisted mainly of alumina with yttrium-rich oxides dispersed throughout, shown in Fig. 4. Isothermal oxidation at $1100{ }^{\circ} \mathrm{C}$, performed in addition to the TCF testing (see Brodin et al. [7] for details), showed that the TGO thickness reached $\sim 10 \mu \mathrm{m}$ after 1000 $\mathrm{h}$ for all specimens. Thus, no significant difference in oxidation rate and oxide composition could be detected. Consequently, specimens with rougher interfaces did not fail prematurely due to $\mathrm{Al}$ depletion. Other studies have found that a rough $\mathrm{BC} / \mathrm{TC}$ interface may lead to faster $\mathrm{Al}$ depletion in the $\mathrm{BC}$ due to the larger area being oxidised [47, 48].

\subsection{Interface roughness}

\subsubsection{Amplitude and spacing parameters}

Fig. 5 shows some of the interface roughness parameters as function of life. $R a$ for the four specimens varied from $6.6 \mu \mathrm{m}$ for specimen R-low to $9.7 \mu \mathrm{m}$ for specimen R-high, see Table 1. It is clear that $R a$, as well as all other amplitude parameters, shown in Fig. 5 a), had a correlation with life. $\mathrm{A} \sim 50 \%$ increase in $R a$, for example, gave a $\sim 70 \%$ increase in TCF life.

$R S m$ does not appear to have a correlation with life as it was quite similar for all specimens, see Fig $5 \mathrm{~b}$ ); the variation in $R S m$ between the specimens was only $\pm 5 \%$ of the mean value of $232 \mu \mathrm{m}$. $R \Delta q$, however, showed a correlation with life similar to that of $R a$. It is notable that different powder sizes used during spraying mainly appeared to influence profile height: the highest $R S m$ value was only about $10 \%$ larger than the lowest, while the corresponding difference in $R a$ was of the order of $50 \%$. Casu et al. [17] found the wavelength to depend on powder size, but that was apparently not the case here.

\subsubsection{Autocorrelation}

The autocorrelation function $(\mathrm{ACF})$ can be used for evaluating the "periodicity' of the interface. Would the $\mathrm{BC} / \mathrm{TC}$ interface be periodic in nature, the ACF can be useful in establishing the wavelength of the profile. Mathematically, the ACF is $\mathscr{F}^{-1}\left\{|\mathscr{F}\{z(x)\}|^{2}\right\}$ where $\mathscr{F}$ denotes Fourier transform. To understand the graphical representation of the ACF, it is enough knowing that a periodic function also has a periodic ACF. 
Fig 6 shows the ACF for a large number of data; the ACF for all specimens had the typical shape of a random profile: the ACF fell rapidly toward zero and had no peaks or valleys. The ACF thus indicated that the studied interface geometries lacked periodicity. The ACF of a cosine wave is also included in Fig. 6 for comparison. The differences between the periodic cosine wave and the specimens' profiles illustrate the lack of periodicity in the profile. Casu et al. [17] successfully used approaches related to the ACF to derive wavelengths from TBC coated specimens. That such an approach has not been successful for the specimens studied here confirms the large process dependence of TBC interface characteristics.

\section{Interface model formulation}

\subsection{Interface models}

The results from the interface roughness measurements were used to create a number of interface models to be used for finite element analysis. Since no inherent periodicity could be found in the interface roughness profile, the suggested interface models will rely on the following assumptions and ideas:

- For convenience, the interface must be modelled as a simple periodic geometry which can be fully described by a small number of parameters, typically amplitude, $A$, and wavelength, $\lambda$.

- A simple periodic geometry will be regarded as representative for the interface if it shares certain 'key roughness parameters' with the actual interface.

- 'Key roughness parameters' are parameters that appear to influence the fatigue life of the TBC system.

\subsubsection{The shape of the interface geometry}

Several tools exist for evaluating the shape of the interface profile, such as the material ratio curve and the profile slope distribution. Fig. 7 shows the slope distribution (in degrees) of the interface profile for the R-low and R-high specimens. Fig. 7 also includes the slope distribution for a cosine wave, an ellipse and a triangular wave; the calculated profile slope distribution was similar to that of an ellipse. For convenience, the slope distribution curve can be transformed into a single numerical parameter by integrating the slope distribution curve from 0 to $25^{\circ}$. This parameter 
will be referred to as $f_{\leqslant 25^{\circ}}$ and should be interpreted as the fraction of the profile with slope $\leqslant 25^{\circ}$. Values of this parameter are listed in Table 1.

Fig. 8 shows the material ratio curves for the specimens with lowest and highest interface roughness. The material ratio curves show the fraction of bond coat in a cross-section of the profile (parallel to the interface) at a given height. The difference in material ratio curves between the specimens is more conveniently quantified by the parameter $R k$, which is explained graphically in Fig. 8. Values of $R k$ are reported in Table 1.

Fig. 8, also contains the results from a cosine wave, an ellipse and a triangular waveform (the cosine and ellipse material ratios have been plotted below the other graphs, not to clutter the plot). It can be seen that the triangular wave, and to some extent also the cosine wave, capture the main characteristics of the profile material ratio curve. The 'tails' at the beginning and the end of the curve, which deviate from the linear behaviour, may be thought of as coming from sparsely dispersed large peaks and valleys which are not considered here.

In addition to the commonly used cosine wave, there appear to be reason for continued evaluation also of elliptical and triangular shapes for use as interface models. In FE modelling, the interface can be modelled as a least representative cell as the first half of the wavelength sufficiently describes the entire interface; the equations for the interface models thus becomes

$$
\begin{aligned}
& z=A \cos \left(\frac{2 \pi}{\lambda} x\right), \quad 0 \leq x \leq \frac{\lambda}{2}, \quad \text { cosine } \\
& z=A \sqrt{1-\left(\frac{4}{\lambda} x\right)^{2}}, \quad 0 \leq x \leq \frac{\lambda}{2}, \quad \text { ellipse } \\
& z=A\left(1-\frac{4}{\lambda} x\right), \quad 0 \leq x \leq \frac{\lambda}{2}, \quad \text { triangle }
\end{aligned}
$$

\subsubsection{Amplitude}

The amplitude can be obtained from amplitude parameters. Fig. 9 a) shows the amplitudes derived from the parameters $R c, R a, R q$ and $R k$ for cosine, ellipse and triangle shapes. For $R c$ and $R k$, the amplitude is calculated as $A=R c / 2$ and $A=R k / 2$. For $R a$ and $R q$, the relation between amplitude and roughness parameter is achieved by entering Eq. 6, 7 and 8 into Eq. 2 and 3, giving 


$$
A=\left\{\begin{array}{lll}
\frac{\pi}{2} R a & \text { or } & \sqrt{2} R q, \text { cosine } \\
\frac{4}{\pi} R a & \text { or } & \sqrt{\frac{3}{2}} R q, \text { ellipse } \\
2 R a & \text { or } & \sqrt{3} R q, \text { triangle }
\end{array}\right.
$$

The variation in the calculated amplitude with chosen parameter and shape is clear from Fig. 9 a); the variation in amplitude was about $\pm 25 \%$ from the mean value for each specimen. For each specimen, the mean value of all calculated amplitudes was quite close to the $R q$-based amplitude for a cosine wave, as indicated in Fig. 9 a), for which reason $R q$ will be used as the basis for amplitude calculation, giving amplitudes as listed in Table 2 .

\subsubsection{Wavelength}

$R S m$, contains the mean profile spacing and may be used directly for wavelength estimation, $\lambda=R S m$. The wavelength can also be obtained from $R \Delta q$ by assuming a profile shape, inserting into Eq. 5 and obtaining a relation between amplitude and wavelength, Eq. 11 shows this for a cosine wave.

The slope distribution curve is similar to the $R \Delta q$ parameter in that it contains both height and spacing information. It can be shown that the relation between the wavelength, $A$ and $f_{\leqslant 25^{\circ}}$ will be

$$
\lambda=\frac{4 A f_{\leqslant 25^{\circ}}}{\tan 25^{\circ} \sqrt{1-f_{\leqslant 25^{\circ}}^{2}}}
$$

for an ellipse (where the 'wavelength' is four times the major axis).

Fig. 9 b) shows a number of calculated wavelengths; it can be seen that most methods gave essentially the same wavelength for all specimen. $R S m$ yielded wavelengths in the order of $230 \mu \mathrm{m}$ whereas all other methods gave wavelengths $\approx 100 \mu \mathrm{m} \pm 3 \%$.

A literature review may provide input to whether the wavelength can be expected to be in the order of $\sim 100 \mu \mathrm{m}$ or $\sim 200 \mu \mathrm{m}$. Fig. 10 shows many amplitudes and wavelengths found in literature for plasma sprayed TBCs [7-11, 14, 18, 20-23, 26-31, 33-36, 38-40]; some were estimates from micrographs while others were parametric studies by FE modelling. As evident from the graph, most interface models fall in the range $20-150 \mu \mathrm{m}$ for wavelength and 5-20 $\mu \mathrm{m}$ for amplitude. A (subjective) estimate of the wavelength to amplitude ratio is $\lambda / A \sim 4-8$, as shown in Fig. 10. Based on 
the calculated amplitudes in Table 2, this would yield wavelengths in the interval $45-165 \mu \mathrm{m}$ for the specimens studied here; $R S m$ was therefore not considered usable for wavelength estimations.

As $R \Delta q$ together with $R q$ for a cosine shape was close to the mean value, see Fig. $9 \mathrm{~b}$ ), it is suggested that the wavelength of any interface could be calculated according to

$$
\lambda=\frac{2 \pi R q}{R \Delta q}
$$

which yields wavelengths of the order of $100 \mu \mathrm{m}$ for all specimens.

\section{Interface model evaluation}

\subsection{Crack modelling}

To evaluate the suggested interface models, crack growth in, or close to, the $\mathrm{BC} / \mathrm{TC}$ interface was modelled using the Abaqus software. The crack was modelled as growing from a peak towards a valley either in the TGO/TC interface, or as initially growing in the TGO/TC interface and then kinking out into the top coat. The used interface models are summarised in Table 2. The finite element analysis was linear elastic with material data from Jinnestrand [49].

It may be argued that cracks growing in an interface grow in a mixed mode [7, 49], which requires both $K_{I}$ and $K_{I I}$ to be calculated. The stress intensity factors, $K_{I}$ and $K_{I I}$, were calculated according to a procedure described elsewhere, see for example Ref. [5, 49, 50]. Crack modelling was performed on a least representative cell, i.e. half of the wavelength. The calculated stress intensity factors are reported here as a function of the damage, $D$, which is defined as the horizontal crack length divided by half the wavelength.

Fig. 11 shows $K_{I}$ and $K_{I I}$ for the three models; the figure shows results from the specimen with highest roughness and a TGO thickness of $6 \mu \mathrm{m}$. Fig. $11 \mathrm{a}$ ) and b) shows the stress intensity factors from a crack growing in the TGO/TC interface. It can be seen that the $K_{I}$ curves, Fig. 11 a), are rather similar for the three models with the cosine model giving $K_{I}$ values in between the ellipse- and triangle-based models. The $K_{I I}$ curves, Fig. 11 b), differ more between the models, but still the cosine model gives values in between those of the ellipse and triangle models.

It is often observed that failure occurs partly in the $\mathrm{BC} / \mathrm{TC}$ interface and partly in the TC. For example, Eriksson et al. [51] found from fractography 
that the failure of a thermally cycled TBC specimen occurred to $\sim 40 \%$ in the $\mathrm{BC} / \mathrm{TC}$ interface and the remainder in the TC. Fig. $11 \mathrm{c}$ ) and d) therefore shows the stress intensity factor for a crack initially growing in the interface and kinking out into the TC at $D=0.4$. Once the crack has kinked out into the TC, all three models essentially gives the same stress intensity factors. It is hence concluded that the choice of model (cosine, ellipse or triangle) may have little influence on the crack modelling in TBC as part of the fracture often occur in the TC. From Fig. $11 \mathrm{c}$ ) and d) it is evident that the cosine model gives results in between those of the ellipse and triangle models and the cosine geometry is therefore suggested as a suitable interface model.

\subsection{Influence of roughness}

Fig. 12 shows a comparison between the specimens with lowest and highest interface roughness; Fig. 12 a) shows $K_{I}$ and $K_{I I}$ for a TGO/TC crack and b) shows $K_{I}$ and $K_{I I}$ for a kink-crack. It can be seen that the stress intensity factors are higher for an interface with high roughness. This contradicts the experimentally observed tendency for a high roughness to give longer life.

The experimentally observed longer life for specimens with high interface roughness may be explained by letting the crack kink out into the TC at an earlier point for specimens with high roughness than for specimen with low interface roughness since kinking out into the TC causes a drop in stress intensity factors. The cross-sections of the specimens studied here show such a tendency: the specimen with lowest interface roughness appears to crack mainly in the interface whereas the other specimen cracks to $\sim 20$ $\%$ in the TC. The exact numbers, however, are somewhat unreliable; the $\mathrm{TC}$, in many cases, still clings on to the $\mathrm{BC}$ and it is not possible to say for sure where the final fracture will occur. The results from Eriksson et al. [51], suggesting the failure to occur to $\sim 40 \%$ in the interface, is considered a more reasonable level of interface fracture. Furthermore, Sjöström et al. [50] has suggested that $K_{I}=\left|K_{I I}\right|$ or $\max \left|K_{I I}\right|$ may be used as criteria for when a crack kinks out into the TC. $K_{I}=\left|K_{I I}\right|$ and $\max \left|K_{I I}\right|$ occurs in the $D=0.3-0.7$ interval, see Fig. 12 a). Fig. 13 illustrates the effect of letting cracks kink at different points: In Fig. 13 a), the crack growing in a rough interface kinks at $D=0.3$ and the one growing in a flatter interface kinks at $D=0.6$. In Fig. $13 \mathrm{~b}$ ), the crack growing in a rough interface kinks at $D=0.6$ and the one growing in a flatter interface follows the interface all the way. In both cases, the level of the stress intensity factors are higher 
for the interface with low roughness during later stages of crack growth as the stress intensity for the rough interface drops when it kinks out into the TC.

To further test the concept, the calculated stress intensity factors were used for life estimation of the TBC. The used life model is Paris law-based and has been described elsewhere, see for example Ref. [5, 7, 49]. The life model predicts damage in the TBC system and the end of the life was considered to occur when the damage reached $85 \%$. The actual numbers output from the model are not valid since the model is not calibrated for this particular case; the cycles to failure have therefore been normalised with the longest calculated life set to 100 cycles.

Fig. 14 show the results from the life prediction model. Fig. 14 a) compares the damage for a TGO/TC crack as function of cycles for the specimens with lowest and highest $R a$; contrary to experimental observations the life of the R-high specimen is considerable shorter than for the R-low specimen. Fig. 14 b) shows the damage development for kink-cracks. It is evident that allowing the cracks to kink at different points makes it possible to capture the experimentally observed tendency for high roughness to promote longer life. Compare, for example, the R-low crack kinking at $D=0.4$ with the R-high crack kinking at $D=0.3$ : the predicted life of the R-high specimen is in that case $\sim 65 \%$ longer than the life of the R-low specimen which agrees well with the experimental observation.

\section{Conclusions}

Three different geometries were tried as interface models for an APS TBC system: cosine, ellipse and triangular. Crack growth modelling using the interface models showed that all studied geometries gave roughly similar stress intensity factors; this was particularly true for a crack partly growing in the interface and partly in the top coat. The cosine geometry gave stress intensity factors in between those of the other models and is therefore suggested to be used for crack growth modelling in TBCs.

None of the tried interface geometries captured the experimentally observed trend for high interface roughness to give longer fatigue life. It was suggested that the longer fatigue life for rough interfaces can be explained by a larger fraction of ceramic top coat fracture.

For modelling of interface geometries in TBCs, the following procedure is recommended. 
- Calculate the amplitude from any suitable amplitude roughness parameter, such as $R q$ or $R a$, obtained from image analysis or from a profilometer measurement on the bond coat prior to top coat deposition. The amplitude, $A$, then becomes

$$
A=\sqrt{2} R q \text { or } \frac{\pi}{2} R a
$$

- If only $R a$ or $R q$ is known, estimate the wavelength, $\lambda$, as 4-8 times the amplitude.

- If $R \Delta q$ (or its arithmetic equivalent $R \Delta a$ ) has been established by image analysis or profilometer measurements, calculate the wavelength as

$$
\lambda=\frac{2 \pi R q}{R \Delta q} \text { or } \frac{2 \pi R a}{R \Delta a}
$$

- Model the bond coat/top coat interface as a cosine wave.

$$
z=A \cos \left(\frac{2 \pi}{\lambda} x\right)
$$

\section{Acknowledgment}

This research has been funded by the Swedish Energy Agency, Siemens Industrial Turbomachinery AB, GKN Aerospace Engine Systems, and the Royal Institute of Technology through the Swedish research program TURBO POWER, the support of which is gratefully acknowledged.

\section{References}

[1] J. DeMasi-Marcin, D. Gupta, Surf. Coat. Technol. 68-69 (1994) 1-9.

[2] H. Evans, M. Taylor, Proc. IMechE 220 (2006) 1-10.

[3] G. Goward, Surf. Coat. Technol. 108-109 (1998) 73-79.

[4] M. Pomeroy, Mater. Des. 26 (2005) 223-231.

[5] S. Sjöström, H. Brodin, in: D. Zhu, H.-T. Lin, S. Mathur, T. Ohji (Eds.), Advanced Ceramic Coatings and Interfaces V, volume 31 of Ceramic Engineering and Science Proceedings, John Wiley \& Sons, Inc., Hoboken, NJ, USA, 2010.

[6] M. Jinnestrand, S. Sjöström, Surf. Coat. Technol. 135 (2001) 188-195. 
[7] H. Brodin, R. Eriksson, S. Johansson, S. Sjöström, in: D. Zhu, H.-T. Lin, D. Singh, J. Salem (Eds.), Advanced Ceramic Coatings and Interfaces IV, volume 30 of $\mathrm{Ce}$ ramic Engineering and Science Proceedings, John Wiley \& Sons, Inc., Hoboken, NJ, USA, 2010, pp. 113-124.

[8] M. Ahrens, R. Vaßen, D. Stöver, Surf. Coat. Technol. 161 (2002) 26-35.

[9] J. Aktaa, K. Sfar, D. Munz, Acta Mater. 53 (2005) 4399-4413.

[10] M. Bäker, J. Rösler, G. Heinze, Acta Mater. 53 (2005) 469-476.

[11] S. N. Basu, G. Ye, R. Khare, B. McCandless, M. Gevelber, D. Wroblewski, International Journal of Refractory Metals \& Hard Materials 27 (2009) 479-484.

[12] T. Beck, M. Bialas, P. Bednarz, L. Singheiser, K. Bobzin, N. Bagcivan, D. Parkot, T. Kashko, I. Petkovic, B. Hallstedt, S. Nemna, J. M. Schneider, Adv. Eng. Mater. 12 (2010) 110-126.

[13] P. Bednarz, Finite element simulation of stress evolution in thermal barrier coating systems, Ph.D. thesis, RWTH Aachen University, 2006.

[14] M. Bialas, Surf. Coat. Technol. 202 (2008) 6002-6010.

[15] E. P. Busso, Z. Q. Qian, M. P. Taylor, H. E. Evans, Acta Mater. 57 (2009) 23492361.

[16] A. Casu, J.-L. Marqués, R. Vaßen, D. Stöver, Key Eng. Mater. 333 (2007) 263-268.

[17] A. Casu, J. Marques, R. Valien, D. Stöver, in: Advanced Ceramic Coatings and Interfaces, volume 27 of Ceramic Engineering and Science Proceedings, pp. 115126.

[18] A. M. Freborg, B. L. Ferguson, W. J. Brindley, G. J. Petrus, Mater. Sci. Eng., A 245 (1998) 182-190.

[19] M. He, J. Hutchinson, A. Evans, Mater. Sci. Eng., A 345 (2003) 172-178.

[20] U. Hermosilla, M. Karunaratne, I. Jones, T. Hyde, R. Thomson, Mater. Sci. Eng., A 513-514 (2009) 302-310.

[21] R. Herzog, N. Warnken, I. Steinbach, B. Hallstedt, C. Walter, J. Müller, D. Hajas, E. Münsterman, J. Schneider, R. Nickel, D. Parkot, K. Bobzin, E. Lugscheider, P. Bednarz, O. Trunova, L. Singheiser, Adv. Eng. Mater. 8 (2006) 535-562.

[22] C.-H. Hsueh, P. Becher, E. Fuller, S. Langer, W. Carter, Mater. Sci. Forum 308-311 (1999) 442-449.

[23] C.-H. Hsueh, J. Haynes, M. Lance, P. Becher, M. Ferber, E. F. Jr, S. Langer, W. Carter, W. Cannon, J. Am. Ceram. Soc. 82 (1999) 1073-1075.

[24] M. Jinnestrand, H. Brodin, Mater. Sci. Eng., A 379 (2004) 45-57.

[25] A. M. Karlsson, Key Eng. Mater. 333 (2007) 155-166.

[26] Y. Liu, C. Persson, J. Wigren, J. Therm. Spray Technol. 13 (2004) 415-424.

[27] Y. Liu, C. Persson, S. Melin, J. Therm. Spray Technol. 13 (2004) 554-560.

[28] Y. Liu, C. Persson, S. Melin, J. Therm. Spray Technol. 13 (2004) 377-380.

[29] Y. Liu, C. Persson, S. Melin, J. Wigren, J. Therm. Spray Technol. 14 (2005) 258263.

[30] M. Martena, D. Botto, P. Fino, S. Sabbadini, M. M. Gola, C. Badini, Eng. Fail. Anal. 13 (2006) 409-426.

[31] G. Petrus, B. Ferguson, J. Therm. Spray Technol. 6 (1997) 29-34.

[32] M.-J. Pindera, J. Aboudi, S. Arnold, Mater. Sci. Eng., A 284 (2000) 158-175.

[33] M. Ranjbar-Far, J. Absi, G. Mariaux, F. Dubois, Mater. Des. 31 (2010) 772-781.

[34] J. Rösler, M. Bäker, K. Aufzug, Acta Mater. 52 (2004) 4809-4817. 
[35] J. Rösler, Adv. Eng. Mater. 7 (2005) 50-54.

[36] K. Sfar, J. Aktaa, D. Munz, Mater. Sci. Eng., A 333 (2002) 351-360.

[37] F. Traeger, M. Ahrens, R. Vaßen, D. Stöver, Mater. Sci. Eng., A 358 (2003) 255-265.

[38] R. Vassen, G. Kerkhoff, D. Stöver, Mater. Sci. Eng., A 303 (2001) 100-109.

[39] X. C. Zhang, B. S. Xu, H. D. Wang, Y. X. Wu, Mater. Des. 27 (2006) 989-996.

[40] C. Zhou, C. Wang, Y. Song, Mater. Sci. Eng., A 490 (2008) 351-358.

[41] E. Gadelmawla, M. Koura, T. Maksoud, I. Elewa, H. Soliman, J. Mater. Process. Technol. 123 (2002) 133-145.

[42] ISO 4287 geometrical product specifications (gps) - surface texture: Profile method - terms, definitions and surface texture parameters, 1997.

[43] ISO 4288 geometrical product specifications (gps) - surface texture: Profile method - rules and procedures for the assessment of surface texture, 1997.

[44] ISO 11562 geometrical product specifications (gps) - surface texture: Profile method - metrological characteristics of phase correct filters, 1996.

[45] R. Eriksson, S. Sjöström, H. Brodin, S. Johansson, L. Östergren, X.-H. Li, Influence of interface roughness on the fatigue life of thermal barrier coatings, to be presented at ICF13 Beijing, China, 2013.

[46] A. Nusair Khan, J. Lu, H. Liao, Surf. Coat. Technol. 168 (2003) 291-299.

[47] C. Che, G. Wu, H. Qi, Z. Huang, X. Yang, Surf. Eng. 24 (2008) 276-279.

[48] N. Czech, M. Juez-Lorenzo, V. Kolarik, W. Stamm, Surf. Coat. Technol. 108-109 (1998) 36-42.

[49] M. Jinnestrand, Delamination in APS applied thermal barrier coatings: life modelling, Ph.D. thesis, Linköpings universitet, 2004.

[50] S. Sjöström, H. Brodin, M. Jinnestrand, Thermomechanical fatigue life of a TBC - comparison of computed and measured behaviour of delamination cracks, to be presented at ICF13 Beijing, China, 2013.

[51] R. Eriksson, H. Brodin, S. Johansson., L. Östergren, X.-H. Li, in: Proceedings of the 19th European Conference on Fracture, ECF19. 


\begin{tabular}{lllllllllllll}
\hline Specimen & TCF & life, & $R a$, & $R q$, & $R c$, & $R p$, & $R v$, & $R S m$, & $R \Delta q$ & $f_{\leqslant 25^{\circ}} R k$, & $R s k$ & $R k u$ \\
& cycles & $\mu \mathrm{m}$ & $\mu \mathrm{m}$ & $\mu \mathrm{m}$ & $\mu \mathrm{m}$ & $\mu \mathrm{m}$ & $\mu \mathrm{m}$ & & & $\mu \mathrm{m}$ & & \\
\hline R-low & 502 & 6.6 & 8.3 & 26.3 & 19.2 & 20.2 & 232 & 0.52 & 0.74 & 18.7 & 0.012 & 2.9 \\
R-medium-1 & 691 & 7.1 & 8.8 & 27.4 & 19.6 & 21.0 & 223 & 0.54 & 0.72 & 20.7 & 0.023 & 2.7 \\
R-medium-2 & 713 & 8.1 & 9.9 & 30.1 & 21.4 & 23.1 & 228 & 0.60 & 0.72 & 23.3 & 0.039 & 2.7 \\
R-high & 850 & 9.7 & 12.0 & 37.2 & 27.0 & 27.8 & 245 & 0.76 & 0.66 & 28.0 & 0.043 & 2.8 \\
\hline
\end{tabular}

Table 1: Values of the interface roughness parameters. For those parameters not defined in the present paper, see for example Gadelmawla et al. [41] for an explanation. 


\begin{tabular}{llll}
\hline Specimen & Model & Amplitude, $\mu \mathrm{m}$ & Wavelength, $\mu \mathrm{m}$ \\
\hline R-low & cosine & 11.7 & 100 \\
R-medium-1 & cosine & 12.5 & 100 \\
R-medium-2 & cosine & 14.1 & 100 \\
R-high & cosine & 17.0 & 100 \\
R-low & ellipse & 10.2 & 100 \\
R-medium-1 & ellipse & 10.8 & 100 \\
R-medium-2 & ellipse & 12.2 & 100 \\
R-high & ellipse & 14.7 & 100 \\
R-low & triangle & 14.4 & 100 \\
R-medium-1 & triangle & 15.3 & 100 \\
R-medium-2 & triangle & 17.2 & 100 \\
R-high & triangle & 20.8 & 100 \\
\hline
\end{tabular}

Table 2: Amplitudes and wavelengths for the three interface models. 


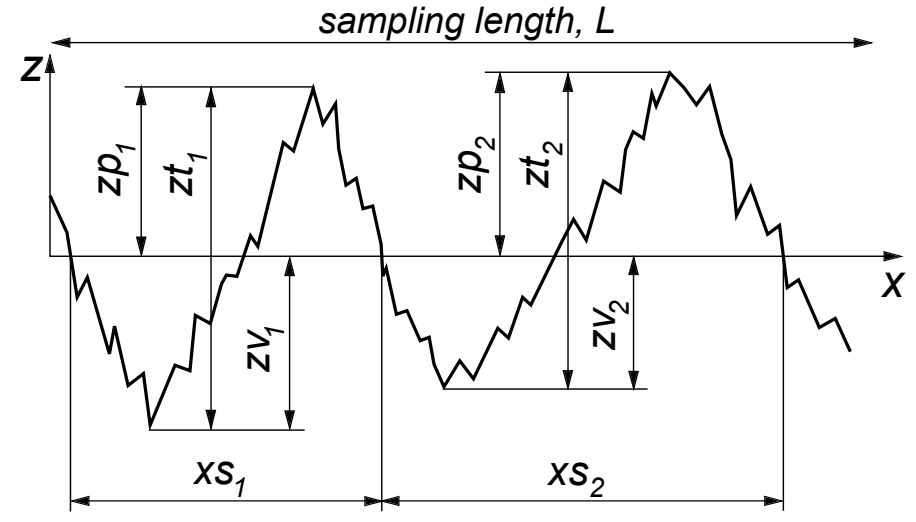

Figure 1: Generic roughness profile. 

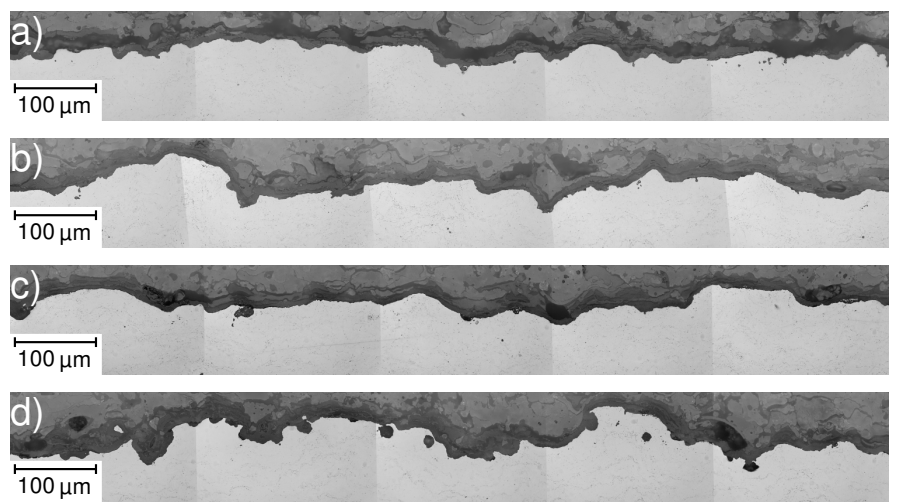

Figure 2: Overview of the interfaces of the four thermally cycled specimens: a)-d) in order of increasing roughness. 


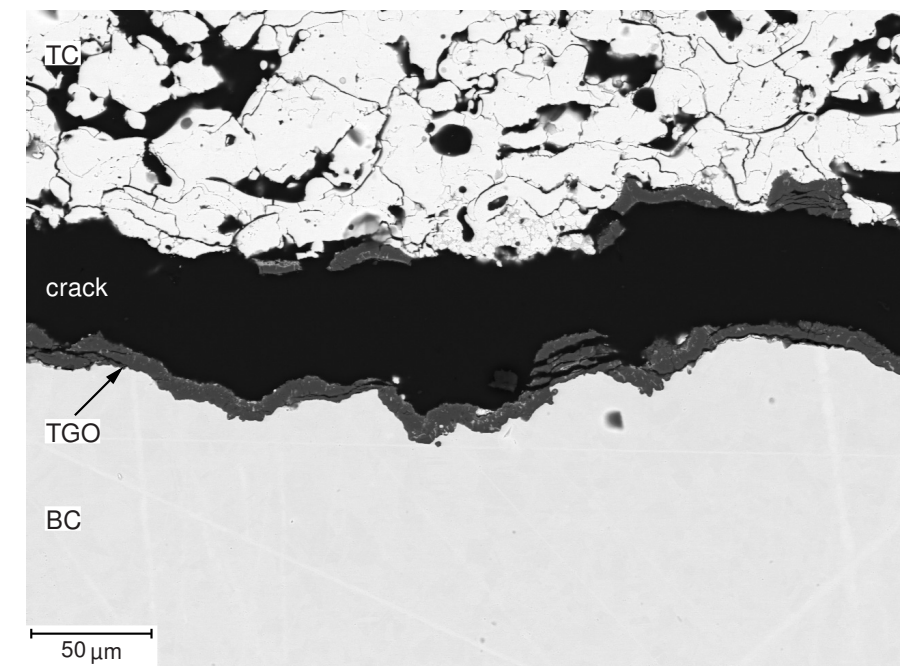

Figure 3: Failed specimen. The failure typically occurs in, or close to, the bond coat/top coat interface. 


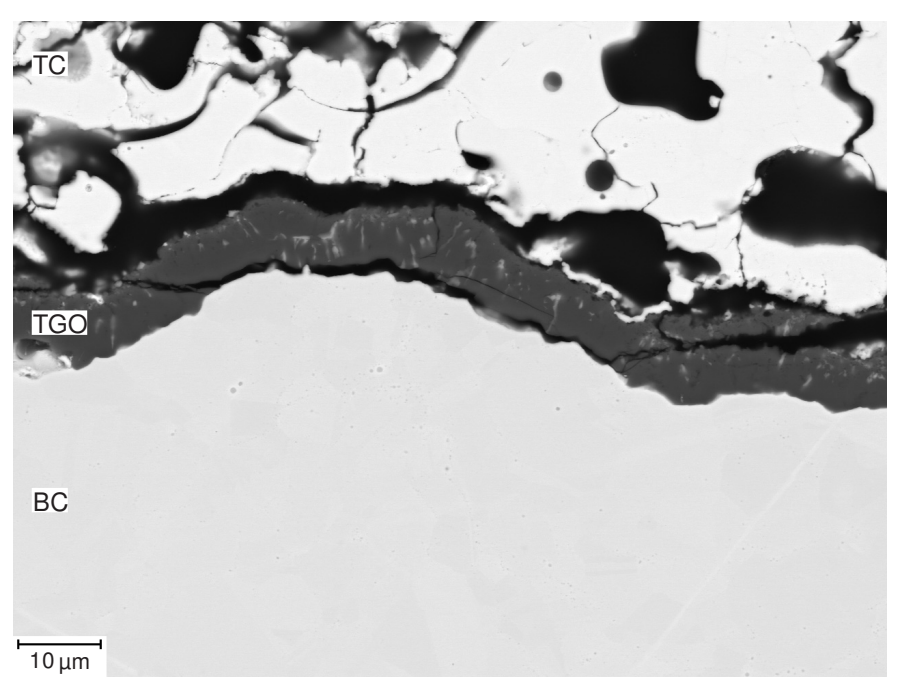

Figure 4: Layer of thermally grown oxides. The oxide layer mainly consisted of alumina with dispersed Y-rich oxides (seen as brighter particles in the dark grey alumina). 
a)

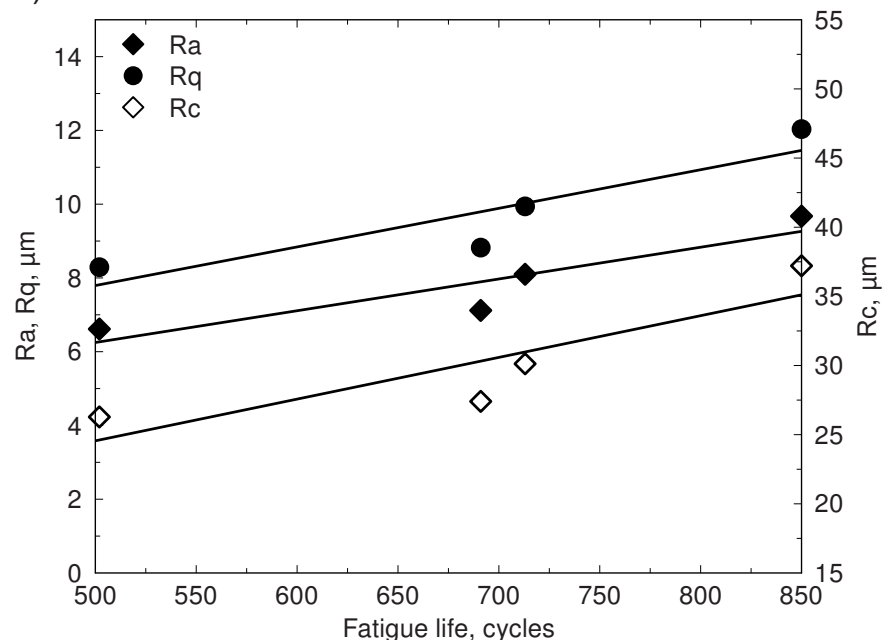

b)

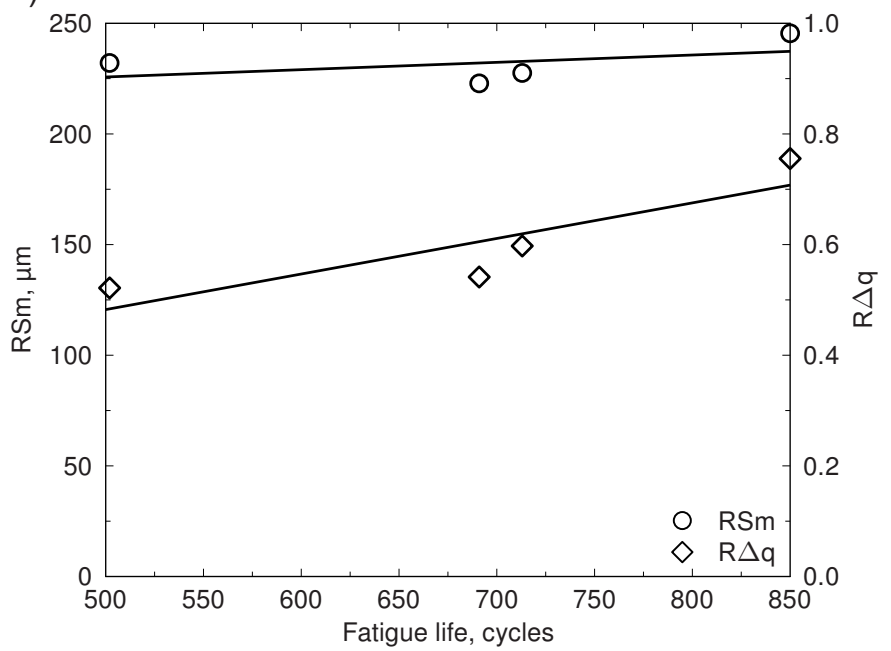

Figure 5: Correlation between interface roughness parameters and fatigue life. a) amplitude parameters b) spacing parameters. 


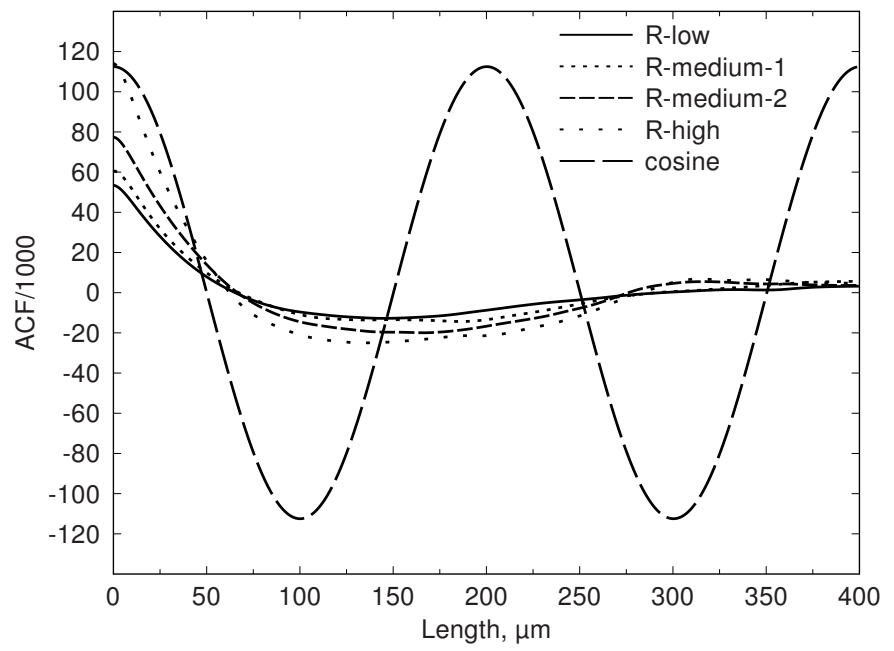

Figure 6: The autocorrelation function (ACF) for the four specimens and a cosine wave. The non-periodic nature of the interface geometries is clear when compared to the cosine wave. 


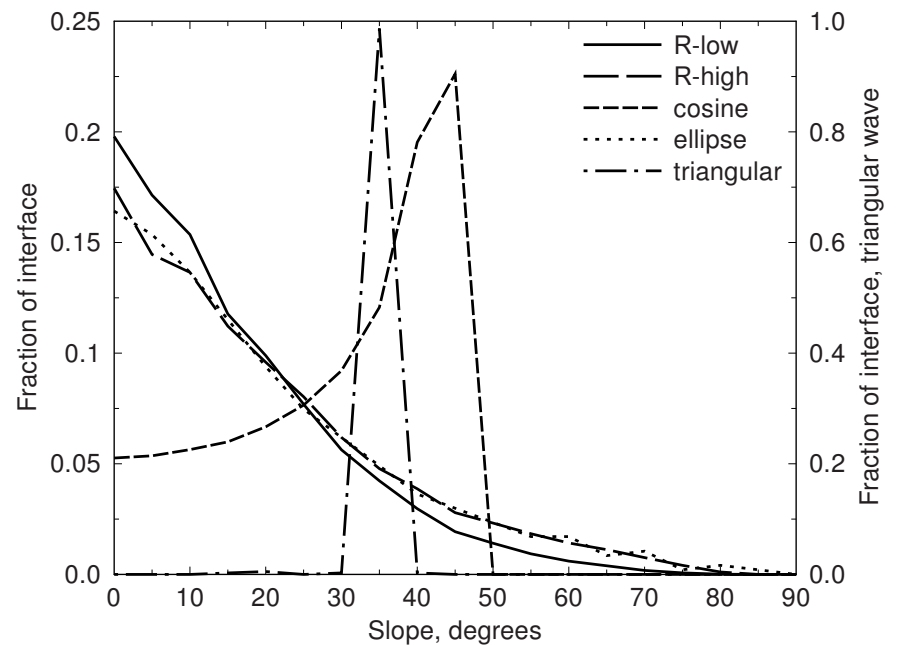

Figure 7: The slope distributions of the specimens with the lowest and highest $R a$. The figure also includes the slope distribution of a cosine, an ellipse and a triangle. 


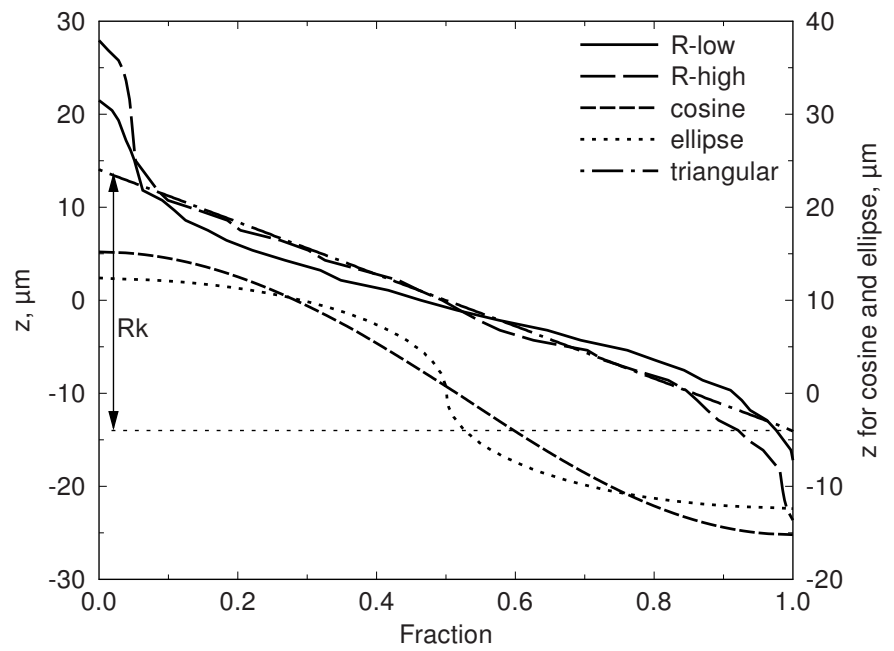

Figure 8: The material ratio curves for the specimens with the lowest and highest $R a$. The figure also includes the material ratio curves of a cosine, an ellipse and a triangle. 

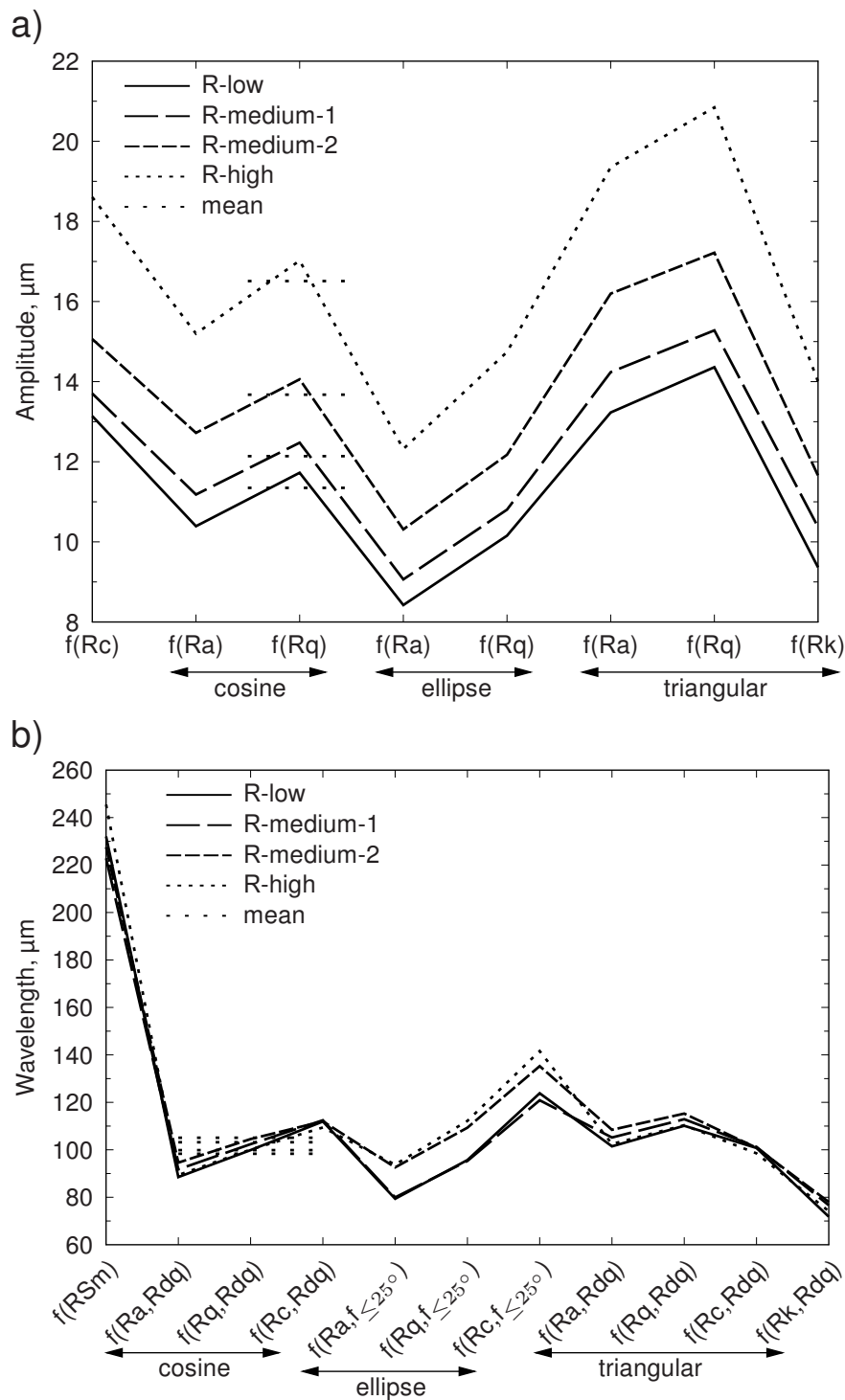

Figure 9: Comparison of amplitudes and wavelengths calculated in different ways: a) amplitudes and b) wavelengths. 


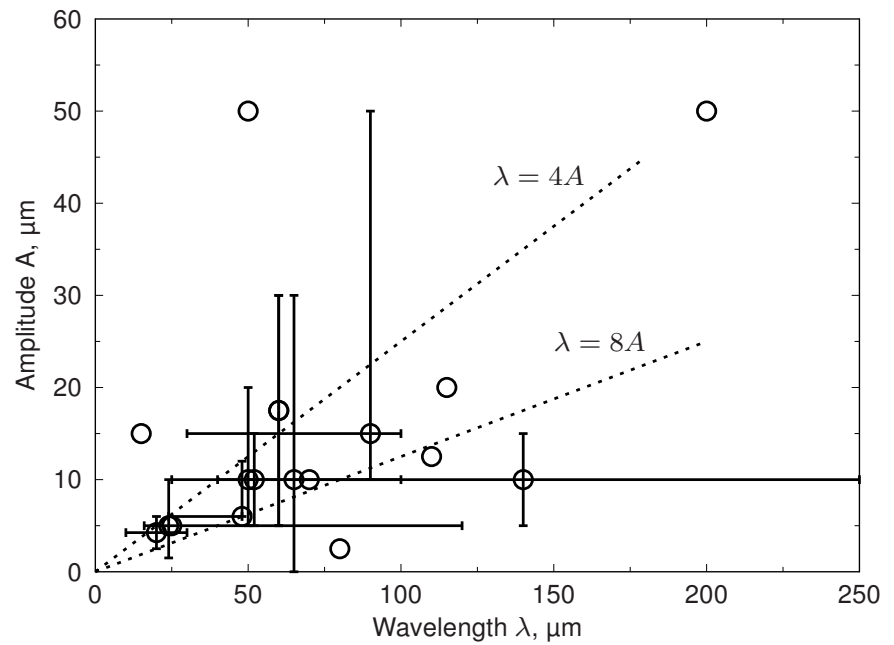

Figure 10: Commonly used values on amplitude and wavelength, from literature. 
a)

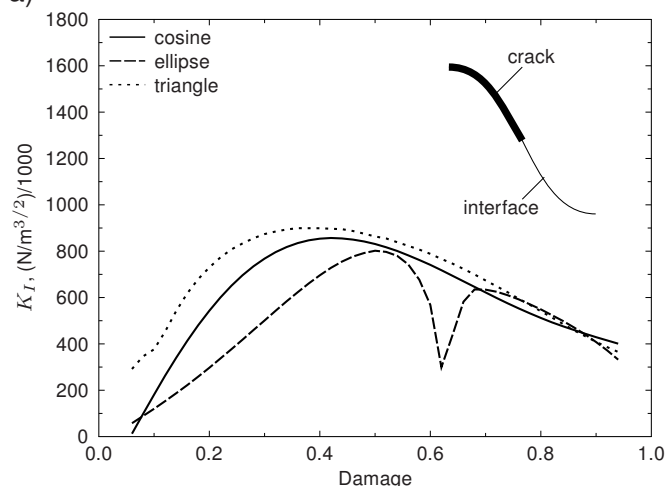

c)

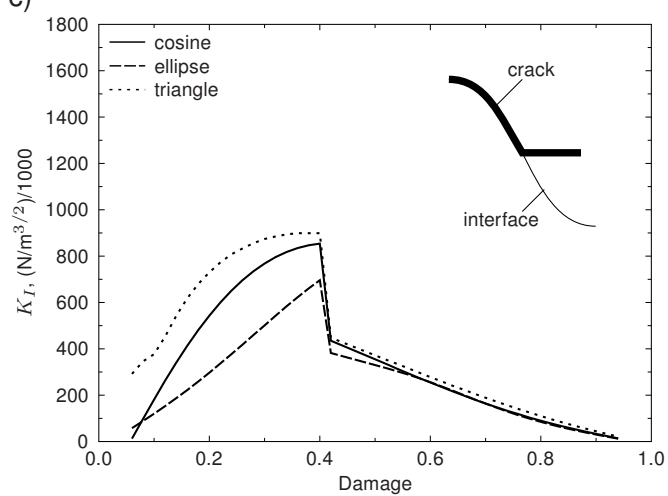

b)

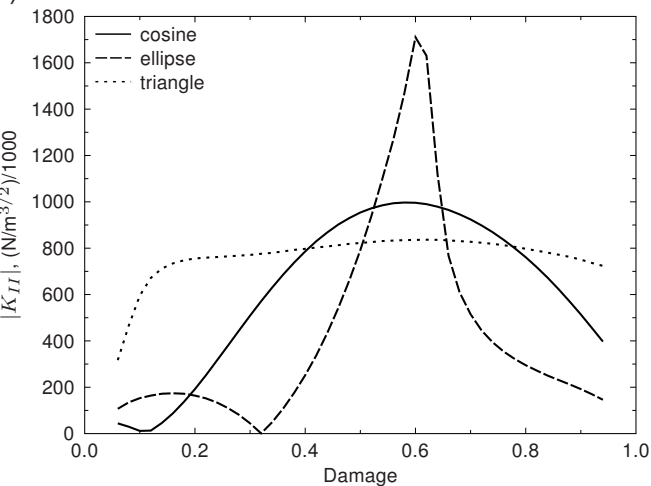

d)

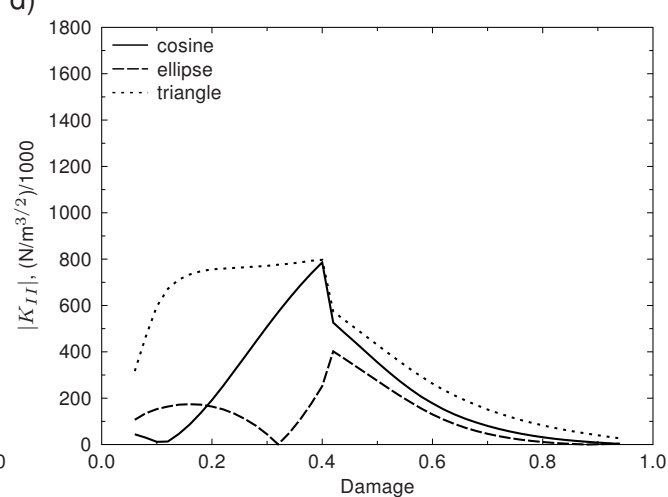

Figure 11: Stress intensity factors, $K_{I}$ and $K_{I I}$, as function of damage. Comparison of different interface geometries for an interface crack: a) $K_{I}$, b) $K_{I I}$. Comparison of different interface geometries for a crack kinking out in the top coat at 0.4 damage: c) $K_{I}$, d) $K_{I I}$. 
a)

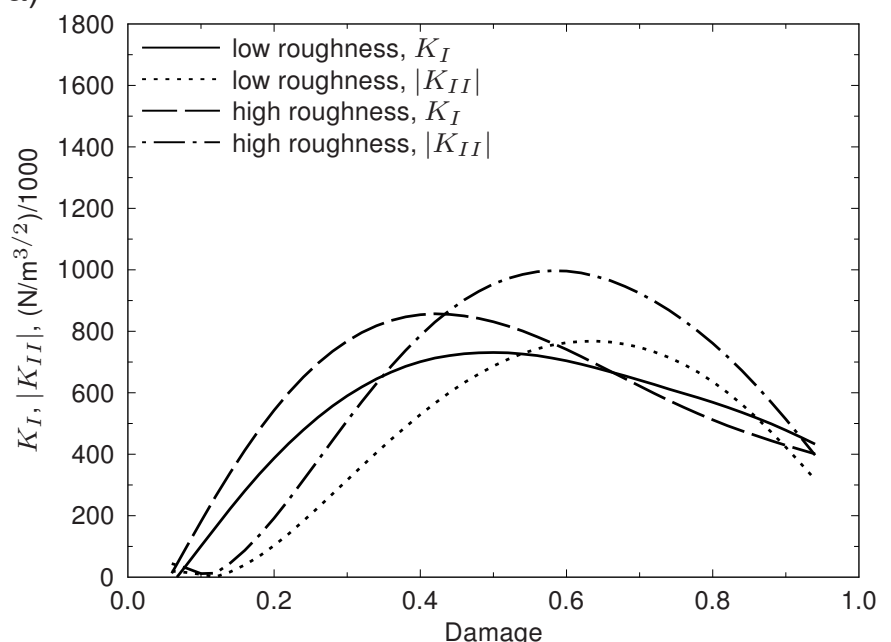

b)

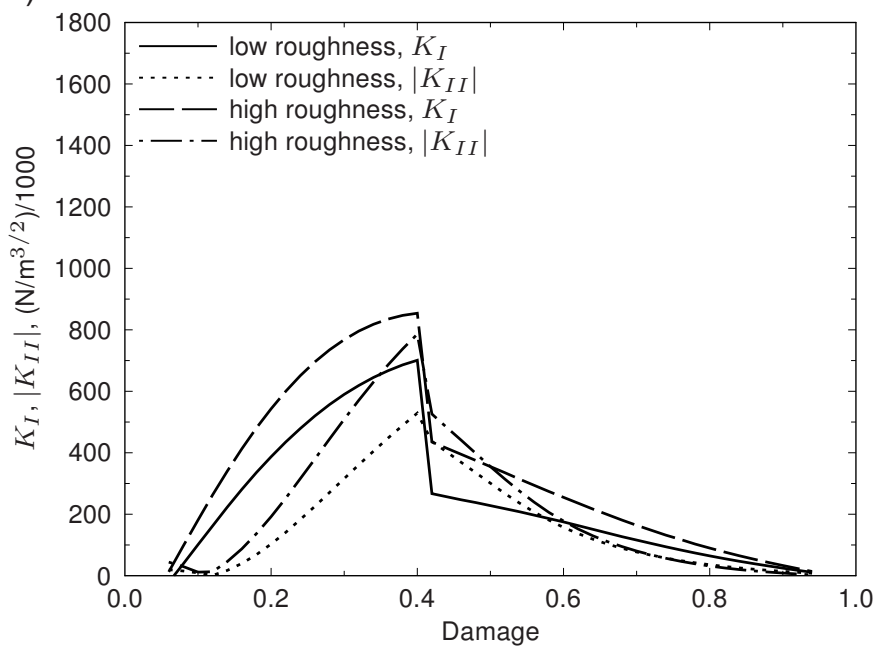

Figure 12: Stress intensity factors, $K_{I}$ and $K_{I I}$, as function of damage. a) Comparison of different roughness for an interface crack. b) Comparison of different roughness for a crack kinking out in the top coat at 0.4 damage. 


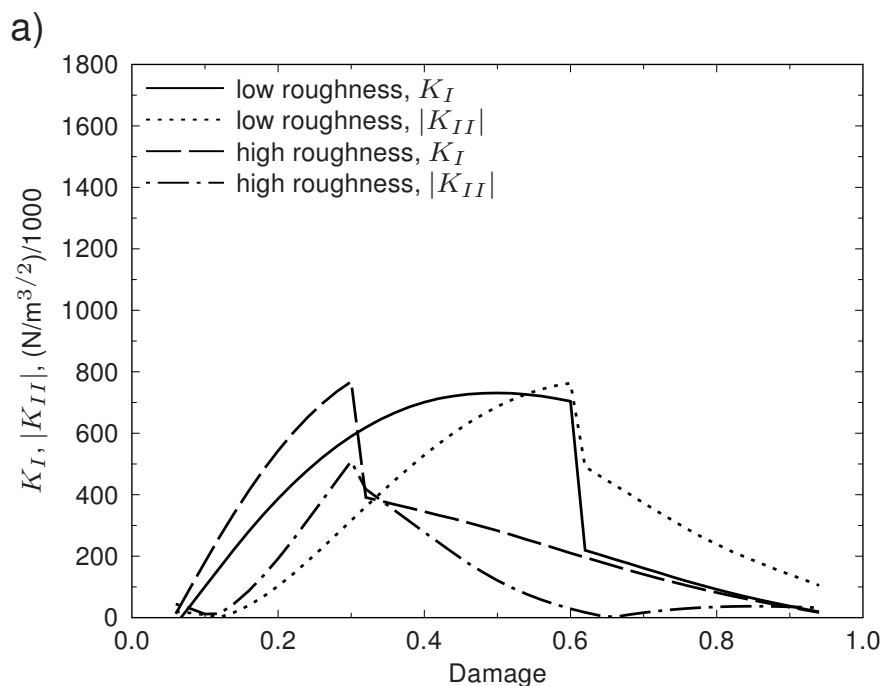

b)

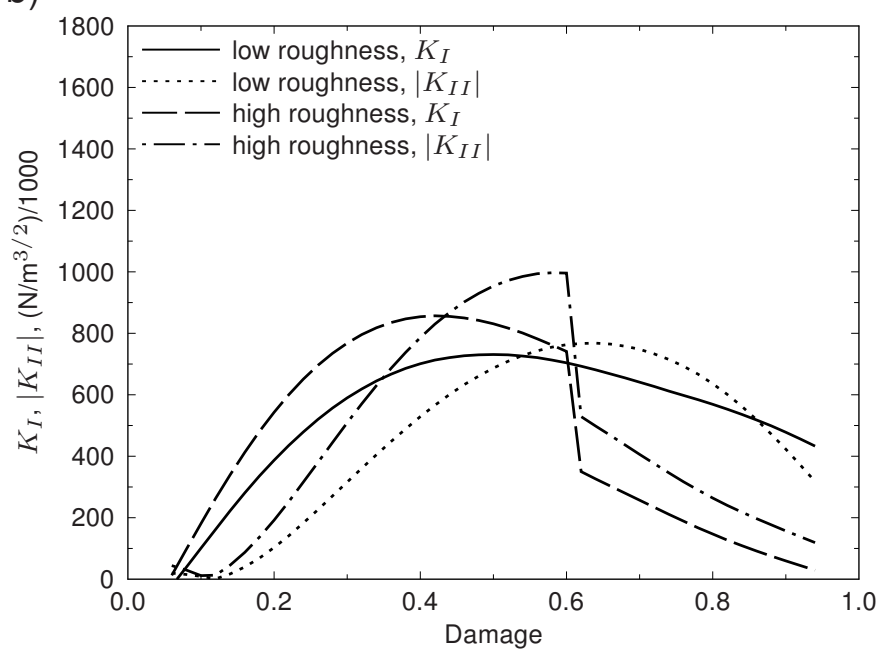

Figure 13: Stress intensity factors, $K_{I}$ and $K_{I I}$, as function of damage for cracks kinking out in the top coat at different points. a) Cracks in a low roughness interface kinking at 0.6 damage and in a high roughness interface kinking at 0.4 damage. b) A crack in a low interface running entirely in the interface and a crack in a high roughness interface kinking at 0.6 damage. 
a)

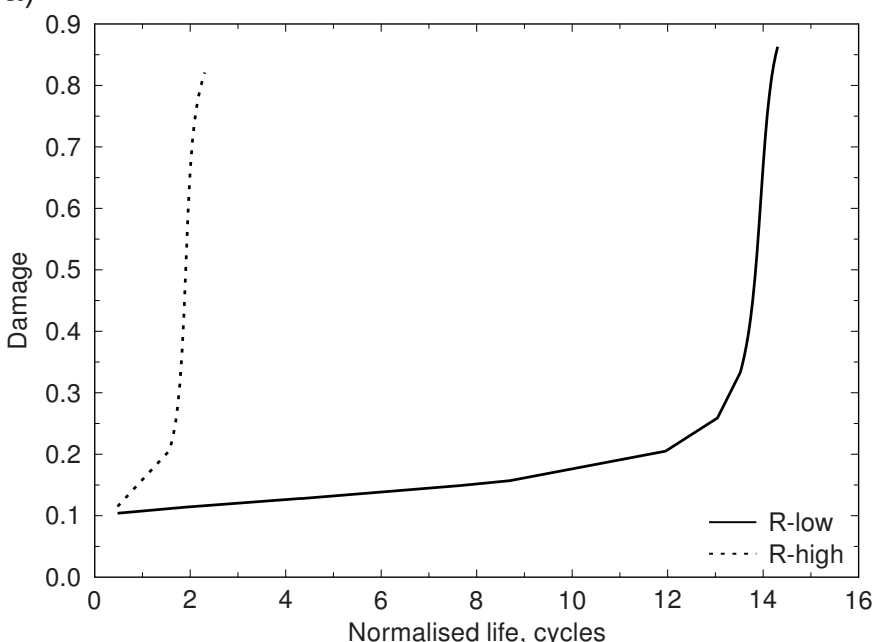

b)

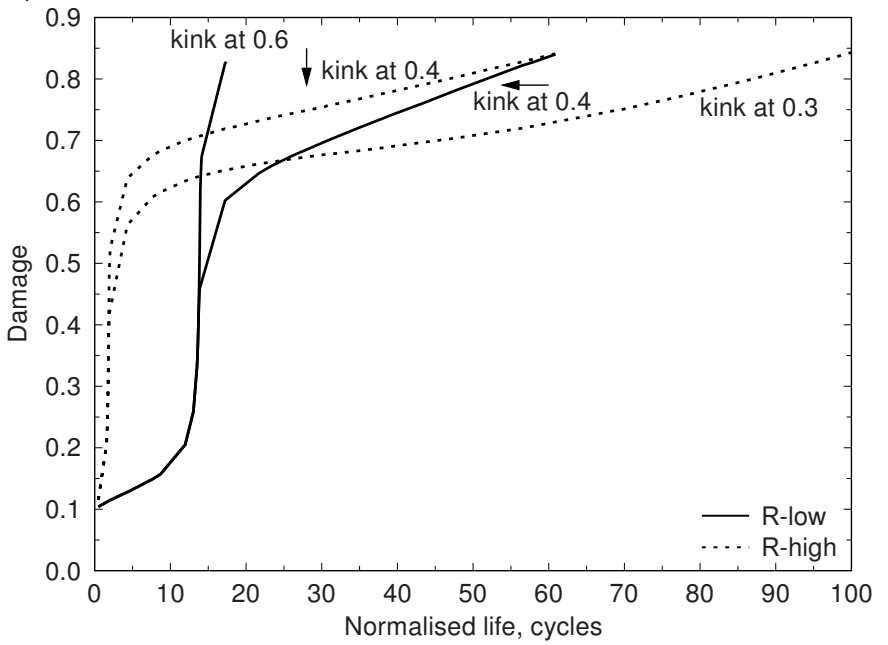

Figure 14: Calculated damage as function of normalised cycles (the longest life was set equal to 100 cycles): a) interface cracks b) comparison of cracks kinking at different positions. 